\title{
Survival Benefit of Metformin Adjuvant Treatment For Pancreatic Cancer Patients: a Systematic Review and Meta-Analysis
}

\author{
Guoxing Wan ${ }^{\mathrm{a}, \mathrm{b}}$ Xue Sun ${ }^{\mathrm{a}}$ Fang Li ${ }^{\mathrm{a}}$ Xuanbin Wang ${ }^{\mathrm{b}, \mathrm{c}}$ Chen Li $\mathrm{i}^{\mathrm{b}, \mathrm{c}}$ \\ Hongliang $\mathrm{Li}^{\mathrm{b}, \mathrm{c}}$ Xianjun $\mathrm{Yu}^{\mathrm{a}}$ Fengjun $\mathrm{CaO}^{\mathrm{a}}$
}

aDepartment of Oncology, Renmin Hospital, Hubei University of Medicine, Shiyan, 'Laboratory of Chinese Herbal Pharmacology, Oncology Center, Renmin Hospital, Hubei University of Medicine, Shiyan, 'Laboratory of Wudang Local Chinese Medicine Research, Hubei University of Medicine, Shiyan, China

\author{
Key Words \\ Metformin • Pancreatic cancer • Therapy • Survival • Meta-analysis
}

\begin{abstract}
Background/Aims: Previous studies on the effect of metformin therapy on survival of pancreatic cancer patients obtained inconsistent findings. To reevaluate the prognostic value of metformin adjuvant treatment, a meta-analysis was carried out. Methods: Relevant articles addressing the association between metformin use and pancreatic cancer survival were electronically searched to identify eligible studies. Pooled hazard ratios (HRs) and $95 \%$ confidence intervals ( $95 \%$ CIs) were calculated to assess the strength of the association. Results: Totally, seventeen studies involving 36791 participants were included. Overall, metformin use was found to be significantly associated with a favorable OS (HR $=0.88,95 \%$ $\mathrm{CI}=0.80-0.97$ ). Subgroup analyses by ethnicity showed a significantly reduced risk of death for metformin users compared with non-users in Asians ( $\mathrm{HR}=0.74,95 \% \mathrm{CI}=0.58-0.94)$ but nonsignificant in Caucasians. When stratified by clinical stage, a remarkable reduction of mortality risk in patients at stage I-II treated with metformin ( $\mathrm{HR}=0.76,95 \% \mathrm{CI}=0.68-0.86)$ was found as well as the group at stage I-IV $(\mathrm{HR}=0.88,95 \% \mathrm{CI}=0.79-0.99)$, but not in patients at stage III-IV. In the stratification analyses based on treatment strategy, metformin therapy was found to be associated with a better clinical outcome in patients receiving surgery or comprehensive therapy ( $\mathrm{HR}=0.73,95 \% \mathrm{CI}=0.62-0.87 ; \mathrm{HR}=0.88,95 \% \mathrm{CI}=0.79-0.97)$ but not chemotherapy. However, the overall analysis failed to show a significant association between metformin use and DFS (HR=1.54, 95\% CI=0.94 -2.50) with only 2 studies enrolled. Conclusion: The current study has evidenced a significant association of metformin adjuvant treatment with the survival benefit for pancreatic cancer patients, suggesting a potentially available option for the treatment. Further investigation is needed.

G. Wan, X. Sun and F. Li contributed equally to this work.

\begin{tabular}{ll}
\hline Fengjun Cao, MD & Department of Oncology, Renmin Hospital, Hubei University of Medicine \\
& Chaoyang Road 39th, Shiyan 442000, Hubei (China) \\
& Tel.+86-0719-8637385; Fax +86-0719-8637385; E-Mail fengjuncaoshiyan@126.com
\end{tabular}
\end{abstract}




\section{Cellular Physiology Cell Physiol Biochem 2018;49:837-847 and Biochemistry \begin{tabular}{l|l} 
DOI: 10.1159/000493214 & (c) 2018 The Author(s). Published by S. Karger AG, Basel \\
Published onlıne: 5 September, 2018 www.karger.com/cpb
\end{tabular} \\ Wan et al.: Metformin and Pancreatic Cancer}

\section{Introduction}

Pancreatic cancer posting the $4^{\text {th }}$ most common cause of cancer-related mortality in the United States accounts approximately for $7 \%$ of cancer death and is one of the most aggressive malignancies with a 5 -year overall survival rate less than $5 \%[1,2]$. Even in patients diagnosed with an early stage and undergoing margin-negative resection, the 5 -year survival rate retains only $24 \%$, and is much worse for patients with unresectable disease [3]. Despite major progress in survival benefit by curative resection over decades, great variability in clinical responses and acquired resistance to chemotherapeutics are more likely to result in tumor recurrence and further progression even for some patients at an early stage with appropriate surgery $[4,5]$, which highlights the need of better treatment strategies for the management of pancreatic cancer, considering the dismal survival data. Of note, the epidemiological investigations find that approximately $80 \%$ of the patients diagnosed with pancreatic cancer are identified with new-onset type 2 diabetes or impaired glucose tolerance, which usually has a negative impact on the survival $[6,7]$. Consequently, the discovery of potential causality between the hypoglycemic action and survival benefit by the clinical studies attaches attention to the underlying mechanisms [8]. Recently, several hypoglycemic agents have been found to be able to reinforce therapeutic effects by targeting the metabolism pathways to inhibit the malignance of cancer cell in addition to the glycemic control for individuals [9]. Of which, metformin exhibits the most promise as an anti-cancer therapy-assistant [10].

Accumulating evidences suggested a preventive role of the well-known anti-diabetic drug metformin accompanied with chemotherapy and radiotherapy in the malignance of cancer cell by inhibiting cell division, promoting apoptosis and autophagy, down-regulating circulating insulin, activating the immune system and crippling irradiation-resistance [8-10]. The preventive role of metformin was shown in multiple cancer types, including pancreatic, colorectal, prostate, and hepatocellular carcinoma, resulting in decreased cancer incidence and mortality $[11,12]$. Particularly in recent years, there are numerous trails with the subject to determine the therapeutic potential of metformin on cancer, frequently demonstrating survival benefit in diabetic cancer patients treated with metformin $[13,14]$. A large prospective observational trial which followed 1300 cancer patients with T2DM who received metformin (289 patients) or another anti-diabetic medication (1064 patients) for 9.6 years showed a $57 \%$ decrease of overall cancer mortality risk for metformin intake [15]. Specially, a recent meta-analysis with 13 observational studies reported that the use of metformin appeared to be associated with a reduced risk of pancreatic cancer in patients with T2DM [16]. Molecularly, it is believed that metformin may primarily exert the antineoplastic activity by activating the serinethreonine liver kinase B (LKB1)/AMP-activated protein kinase (AMPK) and inhibiting the mammalian target of rapamycin (mTOR) signals, which suppresses autophagy but promotes apoptosis of cancer cells [17-19]. Additionally, studies have found that metformin can also suppress insulin-induced tumor growth and angiogenesis through reducing circulating insulin and insulin-like growth factor-1 (IGF1) [20]. Preclinically, metformin was discovered to play a role in the prevention of growth of microscopic foci of metastases after a margin-negative surgical resection [21]. Ongoing research is redefining our understanding about how metformin wields the anti-neoplastic potentials implicated in pancreatic cancer.

Although epidemiologic studies and basic research have repeatedly reported the preventive role of metformin in pancreatic cancer with the decreased cancer incidence and mortality, inconsistent or even conflicting results are obtained by the clinical trails [8], which may result in insufficient confidence to translate these findings to practical utility in the adjuvant setting since benefit of metformin use in the primary prevention or advanced setting may be distinct due to the different mechanisms of action. Moreover, there is still a lack of convincible clinical and epidemiology evidence to support the concerned notion due to the limited randomized controlled trials (RCTs) and perspective studies with large sample size in various populations and a number of issues remain to be properly addressed such 


\section{Cellular Physiology Cell Physiol Biochem 2018;49:837-847 $\begin{array}{lll}\text { DOI: 10.1159/000493214 } & \text { ( } 2018 \text { The Author(s). Published by S. Karger AG, Basel } \\ \text { and Biochemistry } & \text { Published online: } 5 \text { September, } 2018 \text { www.karger.com/cpb }\end{array}$ \\ Wan et al.: Metformin and Pancreatic Cancer}

as at which stage is metformin therapy beneficial, or will different ethnicities or treatment strategies impact on its clinical utility. Therefore, we performed a systematic review and meta-analysis with the current evidence to reassess the clinical significance of metformin therapy in pancreatic cancer patients and to advise further investigations in the future.

\section{Materials and Methods}

\section{Data sources and search strategy}

Electronic databases including PubMed, Embase, Clinical Trials.gov and Cochrane Central Register of Controlled Trials (CENTRAL) were comprehensively searched to retrieve relevant studies on the metformin therapy in pancreatic cancer patients from inception to October 05, 2017 with the limitation of peerreviewed publications reported in English. We developed a search strategy using the following search terms: "metformin" and "pancreatic cancer" to achieve eligible studies. The bibliographies of all relevant studies and review articles were also manually searched to identify additional potentially eligible studies.

\section{Study selection}

Included studies were selected based on the following eligibility criteria: (1) RCTs and nonRCTs(observational, cohort and case-control) investigating the effect of metformin use on the survival of pancreatic cancer patients. (2) studies reporting available time to event data such as hazard ratios (HRs) and $95 \%$ confidence intervals (CIs), Kaplan-Meier curves or relevant information available to calculate the HRs and 95\% CIs. However, studies with a sample size less than 20 or insufficient information, overlapping studies, review and meta-analysis articles and studies pertaining to all cancers but not only pancreatic cancer were excluded. In case of duplication, only the study with the most complete information and largest sample size was selected.

\section{Data extraction and quality assessment}

Retrieved data including first author, year of publication, country, ethnicity of participants, study design, reference, stage, sample size, treatment strategy, adjusted variables, HRs with their $95 \%$ CIs for DFS (diseasefree survival) and OS (overall survival) were independently extracted for all studies into a predesigned table, which were further cross-checked by a second independent reviewer and any discrepancies were resolved by discussion or consultation with a third reviewer until a consensus was reached. Where reported, the HR from adjusted models by potential confounding factors was selected in preference to an unadjusted estimate. In light of only two RCTs included, the quality assessment of included studies was carried out according to the Newcastle-Ottawa quality assessment scale as previously described, concerning methodological quality evaluation including selection, comparability and outcome [22, 23].

\section{Statistical analysis}

HRs with 95\% CIs were directly extracted from the reports of included studies if available, or indirectly estimated from the Kaplan-Meier curves or summary statistics according to the previously described methods [24, 25]. Individual HR with their $95 \%$ CI from each included study was utilized to calculate the pooled effect of metformin therapy on the survival(disease-free survival, DFS including progression-free survival and recurrence-free survival; overall survival, OS) of pancreatic cancer using RevMan 5.2 software (provided by The Cochrane Collaboration, Oxford, UK; http://www.cochrane.org/software/revman.htm), according to the heterogeneity between studies which was measured by the Cochran's $\chi 2$-based $Q$ test and the I-squared test $\left(P<0.10\right.$ or $\mathrm{I}^{2}>50 \%$ was considered indicative of statistically significant heterogeneity). Meanwhile, the significance of pooled HR was evaluated by $\mathrm{Z}$ test ( $P<0.05$ was considered significant) and when the between heterogeneity was significant, the pooled HR was calculated using the fixed effect model, otherwise the random effect model was applied. Also, we preplanned analyses to explore whether the effect of metformin therapy varied according to specific study or patient characteristics, including ethnicities, clinical stages and treatment strategies, which may be the source of between-study heterogeneity as potentially important compounding factors. Moreover, in the current study regarding reference group, the participants who received other hypoglycemic drugs but not metformin were defined as "Nonmetformin", and participants who received neither metformin nor other hypoglycemic drugs were defined as "None", 
while the metformin group was comprised of that participants who had ever used metformin. Furthermore, we conducted the sensitivity analysis by sequential omission of individual studies to assess the stability of pooled results.

\section{Results}

Search results and study characteristics

Initially, a total of 213 articles were retrieved based on the established search strategy. After screening the abstract or full text, 17 eligible studies with 36791 participants with pancreatic cancer were enrolled in this meta-analysis [21, 2641], comprising 15 cohort studies and 2 RCTs. The

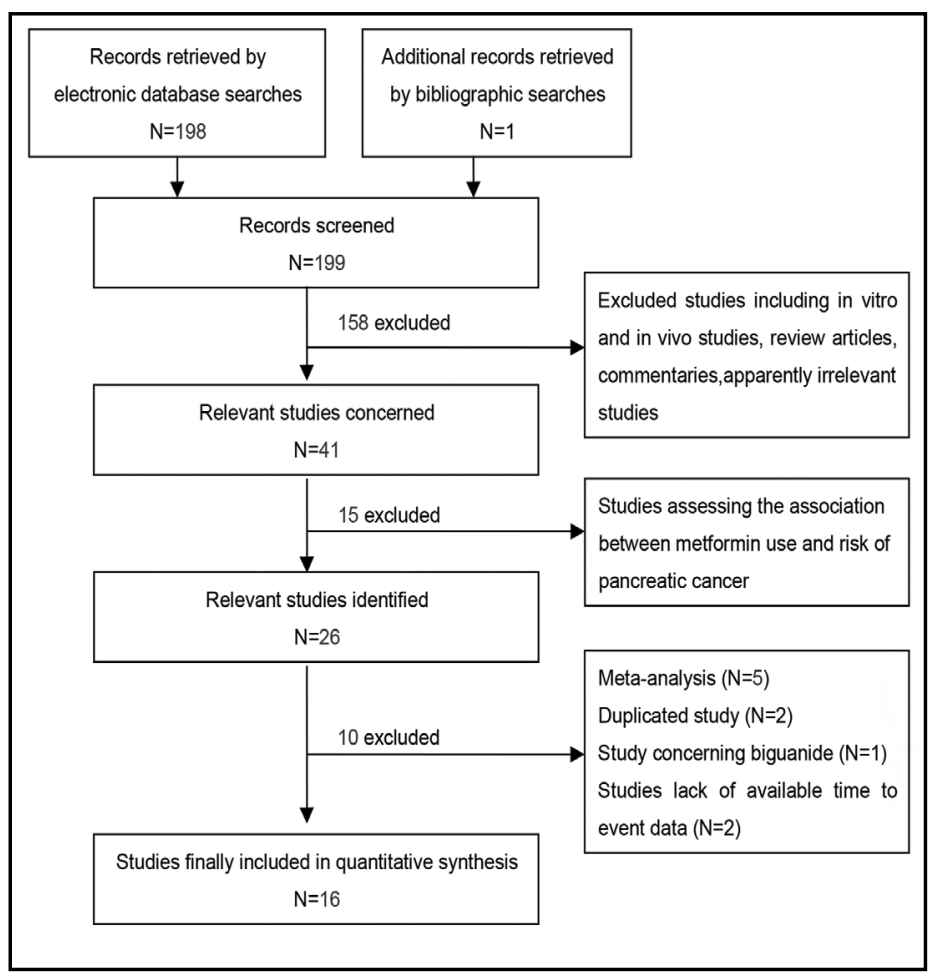

Fig. 1. Flow diagram of study selection for inclusion. participant flow diagram for the included studies is shown in Fig. 1. The sample size of included studies ranged from 44 to 13702 involving I-IV stages and most HRs with 95\%CIs were obtained from multivariable adjusted models. Of which, The HRs with 95\%CIs of 17 studies were estimated for OS and 2 studies for DFS. The main characteristics of included studies were summarized in Table 1 and the quality assessment of included studies was presented in Table 2.

\section{Quantitative synthesis and subgroup analysis}

The pooled analyses for the association between metformin adjuvant treatment and the survival of pancreatic cancer patients were summarized and presented in Table 3 and Fig. 2. Overall, metformin use was found to be significantly associated with a favorable OS (HR=0.88, $95 \% \mathrm{CI}=0.80-0.97, \mathrm{P}=0.01, P_{h}<0.001, \mathrm{I}^{2}=68 \%$ ) by pooling all 17 studies with a random effect model. Subgroup analyses by ethnicity showed a significantly reduced risk of death for metformin users compared with non-users in Asians ( $\mathrm{HR}=0.74,95 \% \mathrm{CI}=0.58-0.94, \mathrm{P}=0.01$ ) but non-significant in Caucasians (HR=0.93, 95\% CI=0.86-1.02, $\mathrm{P}=0.12)$. When stratified by clinical stage, we found a remarkable reduction of mortality risk in patients at stage I-II treated with metformin $(\mathrm{HR}=0.76,95 \% \mathrm{CI}=0.68-0.86, \mathrm{P}<0.0001)$ as well as the group at stage $\mathrm{I}-\mathrm{IV}(\mathrm{HR}=0.88,95 \% \mathrm{CI}=0.79-0.99, \mathrm{P}=0.03)$, but not in patients at III-IV $(\mathrm{HR}=1.08,95 \%$ $\mathrm{CI}=0.82-1.43, \mathrm{P}=0.59$ ). In the stratification analyses based on treatment strategy, metformin therapy was found to be associated with a better clinical outcome in patients receiving surgery or comprehensive therapy(HR=0.73, 95\% $\mathrm{CI}=0.62-0.87, \mathrm{P}=0.0004 ; \mathrm{HR}=0.88,95 \% \mathrm{CI}$ $=0.79-0.97, \mathrm{P}=0.01)$ but not chemotherapy $(\mathrm{HR}=1.11,95 \% \mathrm{CI}=0.76-1.62, \mathrm{P}=0.58)$. However, the overall analysis failed to show a significant association between metformin use and DFS $\left(\mathrm{HR}=1.54,95 \% \mathrm{CI}=0.94-2.50, \mathrm{P}=0.08, P_{h}=0.22, \mathrm{I}^{2}=33 \%\right)$ with only 2 studies enrolled and we therefore were not able to perform subgroup analysis.

\section{Sensitivity analyses}

Considering the large variations across studies, the sensitivity analyses were performed by omitting any single study at a given time to recalculate the pooled HR and $95 \%$ CI. As 


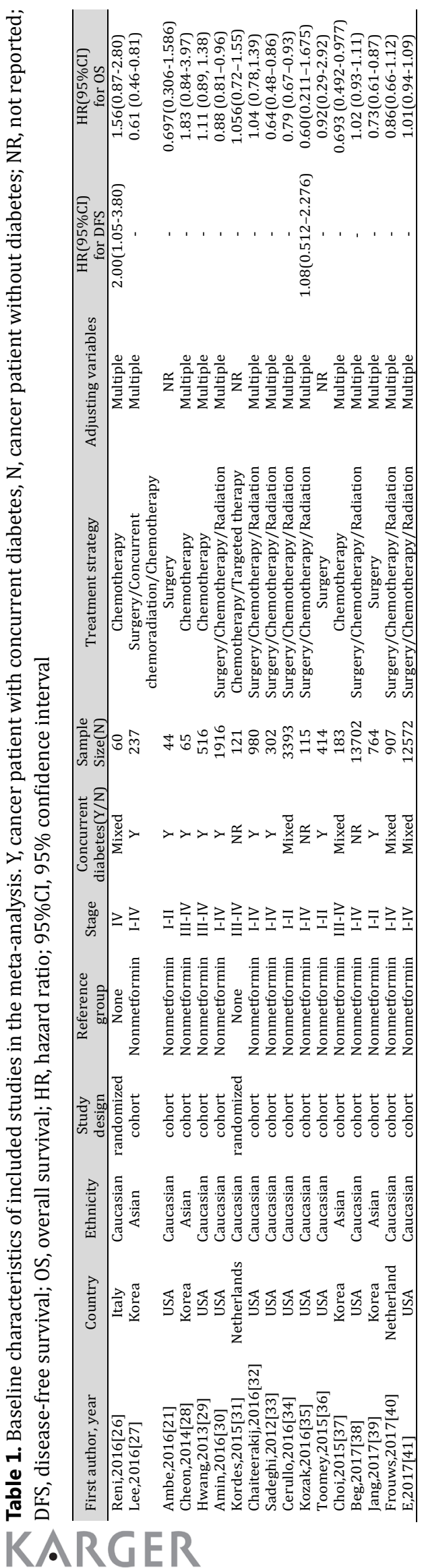

a result, no substantial alteration was found by excluding any specific study, indicating the reliability and robustness of our results, which further lent support to the association between metformin adjuvant treatment and improved survival of pancreatic cancer patients.

\section{Discussion}

The current systematic review and metaanalysis based on 17 studies involving 36791 patients suggests that pancreatic cancer patients with metformin adjuvant treatment may have a prolonged survival compared with those not receiving metformin, though there is evidence of moderate heterogeneity across studies. Specially, the survival benefit may be more evident in patients at an early stage receiving surgery and in Asians.

There is growing consensus that diabetes is a potential cause of pancreatic cancer [10]. Currently, chemotherapy serves as a main supplementation when necessary subsequent to surgery for pancreatic cancer patients at an early stage as well as the primary therapeutic regimen for advanced pancreatic cancer, achieving remarkable benefit, however with evident increase in deleterious effects in relation to the nonspecific damage at the meantime [8]. Thus, it is of highly clinical significance to identify agents which could cooperate with present chemotherapeutic medications to lower the doses without alleviating the effectiveness as well as to avert and/or to surmount drug resistance. Growing evidence has strengthened the role of metformin in prevention and treatment of pancreatic cancer in recent years. Whereas, the variation in the adjuvant effects of metformin administration could be partially interrupted by the differences in tumor stage, treatment strategy or ethnicity originating from both patient characteristics and tumor biology. Recently, two RCTs with 181 advanced pancreatic cancer patients(60 and 121 patients, respectively) conducted in Caucasian population failed to show a survival benefit for metformin users [26, 31]. However, a more recent large cohort study with 764 Asian patients at an early stage(I-II) reported a $27 \%$ decrease of death risk for those receiving metformin adjuvant treatment [39], and similar survival benefit was further confirmed in another lager cohort study (3393 patients) in Caucasian patients who 
Table 2. The quality assessment of the included studies appraised in reference to the Newcastle-Ottawa statement

\begin{tabular}{|c|c|c|c|c|c|c|c|c|c|c|c|}
\hline \multirow{2}{*}{$\begin{array}{l}\text { First author, year } \\
\text { Reni,2016[26] }\end{array}$} & \multirow{2}{*}{$\begin{array}{c}\text { Country } \\
\text { Italy }\end{array}$} & \multicolumn{4}{|c|}{ Selection (4) } & \multicolumn{2}{|c|}{ Comparability (2) } & \multicolumn{3}{|c|}{ Outcome (3) } & \multirow{2}{*}{$\begin{array}{c}\text { Total } \\
8\end{array}$} \\
\hline & & $\Delta$ & $\mathbf{\Delta}$ & $\boldsymbol{\Delta}$ & & $\Delta$ & $\mathbf{\Delta}$ & $\Delta$ & $\boldsymbol{\Delta}$ & $\boldsymbol{\Delta}$ & \\
\hline Lee,2016[27] & Korea & $\Delta$ & $\Delta$ & & & $\boldsymbol{\Delta}$ & $\Delta$ & $\Delta$ & $\boldsymbol{\Delta}$ & $\boldsymbol{\Delta}$ & 7 \\
\hline Ambe,2016[21] & USA & $\Delta$ & $\Delta$ & $\Delta$ & & $\Delta$ & & $\Delta$ & $\Delta$ & & 6 \\
\hline Cheon,2014[28] & Korea & $\Delta$ & $\Delta$ & $\Delta$ & & $\Delta$ & $\Delta$ & $\Delta$ & $\Delta$ & $\Delta$ & 8 \\
\hline Hwang,2013[29] & USA & $\Delta$ & $\Delta$ & $\Delta$ & $\Delta$ & $\Delta$ & $\Delta$ & $\Delta$ & $\Delta$ & $\Delta$ & 9 \\
\hline Amin,2016[30] & USA & $\Delta$ & $\Delta$ & $\Delta$ & & $\Delta$ & $\Delta$ & $\Delta$ & $\boldsymbol{\Delta}$ & $\Delta$ & 8 \\
\hline Kordes,2015[31] & Netherlands & $\Delta$ & $\Delta$ & $\Delta$ & $\Delta$ & $\boldsymbol{\Delta}$ & $\bar{\Delta}$ & $\Delta$ & $\boldsymbol{\Delta}$ & $\Delta$ & 9 \\
\hline Chaiteerakij,2016[32] & USA & $\Delta$ & $\Delta$ & $\Delta$ & $\Delta$ & $\Delta$ & & $\Delta$ & $\Delta$ & $\Delta$ & 8 \\
\hline Sadeghi,2012 [33] & USA & $\Delta$ & $\Delta$ & $\Delta$ & & $\Delta$ & & $\Delta$ & $\Delta$ & & 6 \\
\hline Cerullo,2016[34] & USA & $\Delta$ & $\Delta$ & $\Delta$ & $\Delta$ & $\Delta$ & & $\Delta$ & $\boldsymbol{\Delta}$ & & 7 \\
\hline Kozak,2016[35] & USA & $\Delta$ & $\Delta$ & $\Delta$ & & $\Delta$ & & $\Delta$ & $\mathbf{\Delta}$ & $\Delta$ & 7 \\
\hline Toomey,2015[36] & USA & $\Delta$ & $\Delta$ & & & $\boldsymbol{\Delta}$ & & $\Delta$ & $\boldsymbol{\Delta}$ & & 5 \\
\hline Choi,2015[37] & Korea & $\bar{\Delta}$ & $\bar{\Delta}$ & $\Delta$ & & $\Delta$ & $\Delta$ & $\bar{\Delta}$ & $\bar{\Delta}$ & $\Delta$ & 8 \\
\hline Beg,2017[38] & USA & $\Delta$ & $\Delta$ & & & $\Delta$ & $\Delta$ & $\Delta$ & $\Delta$ & $\Delta$ & 7 \\
\hline Jang,2017[39] & Korea & $\bar{\Delta}$ & $\bar{\Delta}$ & $\Delta$ & & $\bar{\Delta}$ & $\bar{\Delta}$ & $\bar{\Delta}$ & $\bar{\Delta}$ & $\bar{\Delta}$ & 8 \\
\hline Frouws,2017[40] & Netherlands & $\Delta$ & $\Delta$ & $\Delta$ & & $\Delta$ & $\Delta$ & $\Delta$ & $\Delta$ & & 7 \\
\hline $\mathrm{E}, 2017[41]$ & USA & $\bar{\Delta}$ & $\bar{\Delta}$ & $\bar{\Delta}$ & & $\bar{\Delta}$ & $\bar{\Delta}$ & $\bar{\Delta}$ & $\overline{\mathbf{A}}$ & & 7 \\
\hline
\end{tabular}

Table 3. Results of the association between metformin adjuvant treatment and prognosis of pancreatic cancer. $P_{h^{\prime}}$ p value of the $Q$ test for heterogeneity. $R$, random effect model; $F$, fixed effect model

\begin{tabular}{|c|c|c|c|c|c|c|c|c|}
\hline \multirow[t]{2}{*}{ Survival } & \multirow{2}{*}{\multicolumn{2}{|c|}{ Subgroup }} & \multirow{2}{*}{$\begin{array}{c}\text { Number of } \\
\text { studies }\end{array}$} & \multirow{2}{*}{$\begin{array}{c}\text { Pooled analyses } \\
\text { HR(95\%CI) }\end{array}$} & \multirow[t]{2}{*}{$\mathrm{P}$ value } & \multicolumn{2}{|c|}{ heterogeneity } & \multirow{2}{*}{$\begin{array}{l}\text { Effect } \\
\text { model }\end{array}$} \\
\hline & & & & & & $\mathrm{P}_{\mathrm{h}}$ & $\mathrm{I}^{2}$ & \\
\hline \multirow[t]{9}{*}{ OS } & \multicolumn{2}{|c|}{ Overall } & 17 & $0.88[0.80,0.97]$ & 0.01 & $<0.001$ & $68 \%$ & $\mathrm{R}$ \\
\hline & \multirow{3}{*}{ Ethnicity } & Asian & 4 & $0.74[0.58,0.94]$ & 0.01 & 0.08 & $56 \%$ & $\mathrm{R}$ \\
\hline & & Caucasian & 13 & $0.93[0.86,1.02]$ & 0.12 & 0.006 & $56 \%$ & $\mathrm{R}$ \\
\hline & & I-II & 4 & $0.76[0.68,0.86]$ & $<0.0001$ & 0.91 & $0 \%$ & $\mathrm{~F}$ \\
\hline & \multirow[t]{2}{*}{ Stage } & III-IV & 5 & $1.08[0.82,1.43]$ & 0.59 & 0.59 & $58 \%$ & $\mathrm{R}$ \\
\hline & & I-IV & 8 & $0.88[0.79,0.99]]$ & 0.03 & $<0.001$ & $74 \%$ & $\mathrm{R}$ \\
\hline & \multirow{3}{*}{ Treatment strategy } & Surgery & 3 & $0.73[0.62,0.87]$ & 0.0004 & 0.92 & $0 \%$ & $\mathrm{~F}$ \\
\hline & & \multirow{2}{*}{$\begin{array}{c}\text { Chemotherapy } \\
\text { Comprehensive } \\
\text { therapy }\end{array}$} & 4 & $1.11[0.76,1.62]$ & 0.58 & 0.02 & $69 \%$ & $\mathrm{R}$ \\
\hline & & & 10 & $0.88[0.79,0.97]$ & 0.01 & $<0.001$ & $71 \%$ & $\mathrm{R}$ \\
\hline DFS & \multicolumn{2}{|c|}{ Overall } & 2 & $1.54[0.94,2.50]$ & 0.08 & 0.22 & $33 \%$ & $\mathrm{~F}$ \\
\hline
\end{tabular}

received resection and metformin therapy [34]. Nevertheless, there is a lack of convincing evidence in attempt to address the inconsistent findings with appreciate approach.

To our knowledge, the present study was the most comprehensive meta-analysis with the most included acrticles regarding the effect of metformin adjuvant treatment on pancreatic cancer patients with a relatively large sample size, demonstrating a significant survival benefit, in agreement with the previous meta-analyses [42-44]. Zhang et al. demonstrated a trend to improve the prognosis of pancreatic cancer patients with concurrent type 2 diabetes treated with metformin ( $\mathrm{HR}=0.80,95 \% \mathrm{CI}=0.62-1.03)$ by a meta-analysis with 4 publications (1,429 patients) [45], while their meta-analysis with evident between-study heterogeneity failed to show a significant association, which may suggest the significance of sample size when interpreting their results compared with that in the current study. Moreover, the pooling analysis by Zhang et al. indicated that metformin may improve the survival for cancer patients with concurrent diabetes, particularly for breast, colorectal, ovarian, and endometrial cancer, but they failed to show such survival benefit in pancreatic cancer with only 3 studies included ( $\mathrm{HR}=0.80,95 \% \mathrm{CI}=0.62-1.03)$ [14]. Additionally, a recent meta-analysis combining 27 studies comprising 24178 participants was conducted to qualify the adjuvant effect of metformin in cancers, suggesting a better survival, particularly in colorectal and prostate cancer, however without special attention to pancreatic cancer 


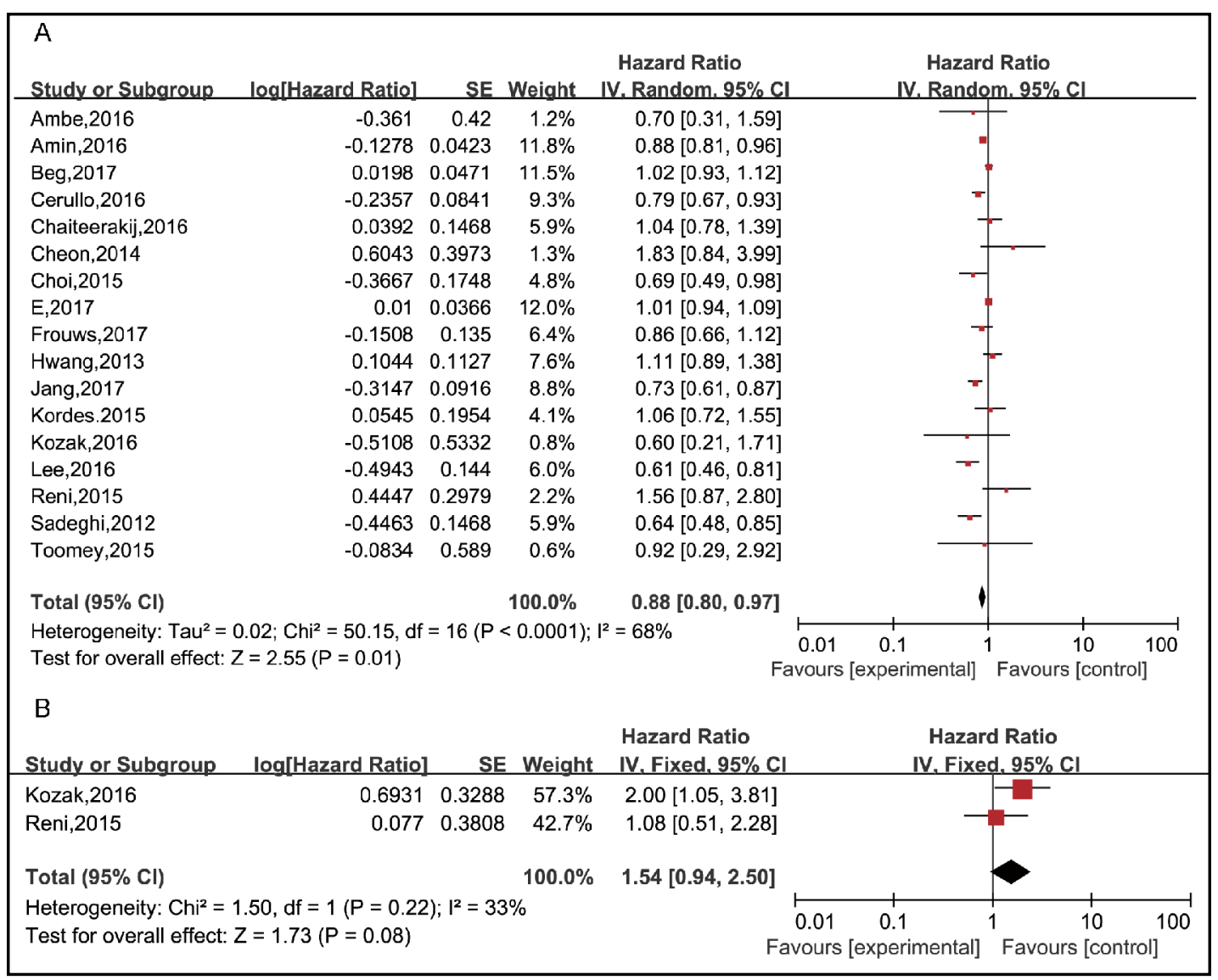

Fig. 2. Meta-analysis results of the effect of metformin therapy on the prognosis of pancreatic cancer patients. A. OS, overall survival. B. DFS, disease-free survival.

[13]. More recently, in spite of less sample sizes, two meta-analyses by Zhou et al. (10 studies with only 9318 participants) [46] and by E et al. (6 studies with 12,057 participants) have represented similar findings with demonstrating a survival benefit of metformin therapy for pancreatic cancer patients [47]. Furthermore, the subgroup analyses based on ethnicity in the current study revealed a more favorable prognosis for metformin therapy in Asian population ( $\mathrm{HR}=0.74, \mathrm{P}=0.01$ ) but not evident in Caucasian population ( $\mathrm{HR}=0.92, \mathrm{P}=0.10)$ which was in line with the findings by Zhou et al., indicating that ethnicity variation may contribute to the metformin action, however, the underlying mechanism for the discrepancy remains undefined. In addition, in the stratification analyses by clinical stage, such survival benefit by metformin adjuvant treatment was also obviously observed in patients at an early stage (I-II) and a mixed stage (I-IV) but not in advanced stage. Meanwhile, similar survival benefit was presented both in patients undergoing surgery and in patients receiving a comprehensive therapy when stratified by treatment strategy, while which did not appear in patients adopting chemotherapy. Notably, as the guideline recommends, more patients at an early stage usually receive surgery treatment but chemotherapy in the advanced stage in the current study, suggesting the potential importance of metformin action following surgery at an early stage. However, few studies have investigated the therapeutic effects of metformin for pancreatic cancer at different stages and the distinct role and advantages of metformin therapy in combination with various treatment strategies. Besides, the possibility that patients who possess the opportunity to have surgery are more likely to situate at an early cancer stage and would thereby face less side effects caused by chemotherapy/radiation should not be neglected as well. Therefore, the underlying mechanisms including cancer stage-dependent effect under specific treatment strategies involved in the findings are 


\section{Cellular Physiology Cell Physiol Biochem 2018;49:837-847 \begin{tabular}{ll|l} 
DOI: $10.1159 / 000493214$ & ( 2018 The Author(s). Published by S. Karger AG, Basel \\
and Biochemistry & Published online: 5 September, 2018 www.karger.com/cpb
\end{tabular} \\ Wan et al.: Metformin and Pancreatic Cancer}

warranted to be determined. Collectively, the present study with more power which was comparatively considered more convincible and reliable supported a favor for metformin adjuvant treatment in pancreatic cancer patients, particularly in patients at an early stage following surgery treatment.

Caution is required for the appropriate interpretation of our results in light of several limitations. Firstly, the inherent weaknesses of non-RCTs data including potential measurement errors in the exposure to metformin, and variation in the definition of metformin use, and the risk of time-related bias may have impacts on the effect of metformin in pancreatic cancer [48]. Secondly, the current study enrolling participants regardless of the diabetes mellitus status and duration may hardly rule out completely the possibility of immortal bias due to the lack of relevant information mentioned above. For instance, diabetic patients without diabetic medication are expected to be healthier than patients on metformin and correspondingly, more advanced diabetic patients who are more likely to receive nonmetformin treatment may decrease the opportunity to have surgery due to expected risk of postoperative complications, therefore, the survival outcomes may be biased by diabetesrelated mortality. Similarly, worse outcomes in patients who used antidiabetic treatment options other than metformin may have more advanced diabetes and for this reason, rather than not using metformin, have worse outcomes compared to patients with metformin therapy. Thirdly, details including metformin dose along with other important factors related to prognosis such as tobacco use, adverse events, cytotoxicity were incomplete, which may result in evidence of heterogeneity and decrease the effectiveness of subgroup analyses accordingly [11]. Fourthly, it would be an over-simplifcation to attribute the survival benefit solely to metformin exposure. As well known, some other hypoglycemic agents such as thiazolidinedione is potentially capable of reducing the incidence of malignancies [49], which therefore may be reasonable to favor the prognosis of pancreatic cancer. In addition, diabetic cancer patients treated with more hypoglycemic agents are quite universal in clinical practice. Consequently, it is hard to determine the specific clinical significance of metformin therapy without eliminating potential anti-cancer action from other hypoglycemic agents. Fifthly, though a significant association of metformin use with better survival outcome was particularly observed in Asians, caution is called for the application of the findings since Asian population is retrieved only from Korea, which would possibly limit the representativeness of conclusion. Meanwhile, different health care systems and medical guidelines for diabetes and pancreatic cancer among populations may affect the use of metformin, which may also contribute to the ethnicity variation effect in addition to the descent of study participants. Sixthly, the superior concern to healthcare or glycemic control may be of importance for the clinical outcome of pancreatic cancer patients particularly for those with concurrent diabetes, however, relevant information was not detailed in the included studies, which prompted future studies to focus it. Lastly, most of the current studies focused on the effect of metformin use in patients with concurrent diabetes, yet it remains unclear that whether metformin will be universally efficacious in pancreatic cancer with clinical and biological heterogeneity or more effective in diabetic patients.

\section{Conclusion}

In conclusion, the current study has evidenced a significant association of metformin adjuvant treatment with survival benefit for pancreatic cancer patients, suggesting a potentially available option for the treatment. Further prospective studies and RCTs are warranted to confirm the findings with the consideration of some confounding variables including diabetes status and duration, dose of metformin use, the influence of other hypoglycemic agents, ethnicity variation as well as other clinical characteristics. 


\section{Cellular Physiology Cell Physiol Biochem 2018;49:837-847

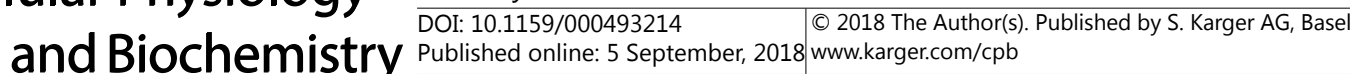

Wan et al.: Metformin and Pancreatic Cancer

\section{Abbreviations}

HR (hazard ratio); 95\% CI (95\% confidence interval); T2DM (Type 2 diabetes mellitus); OS (overall survival); DFS (disease-free survival); RCT (randomized controlled trial).

\section{Acknowledgements}

This work was supported by grants from the Guidance Project of Education Department of Hubei Province (B2016139).

\section{Disclosure Statement}

The authors declare no potential Disclosure Statements.

\section{References}

1 Wolfgang CL, Herman JM, Laheru DA, Klein AP, Erdek MA, Fishman EK, Hruban RH: Recent progress in pancreatic cancer. CA Cancer J Clin 2013;63:318-348.

-2 Siegel R, Desantis C, Jemal A: Colorectal cancer statistics, 2014. CA Cancer J Clin 2014;64:104-117.

3 Ryan DP, Hong TS, Bardeesy N: Pancreatic adenocarcinoma. N Engl J Med 2014;371:1039-1049.

-4 Polyak K, Weinberg RA: Transitions between epithelial and mesenchymal states: acquisition of malignant and stem cell traits. Nat Rev Cancer 2009;9:265-273.

-5 Ailles LE, Weissman IL: Cancer stem cells in solid tumors. Curr Opin Biotechnol 2007;18:460-466.

6 Pannala R, Basu A, Petersen GM, Chari ST: New-onset diabetes: a potential clue to the early diagnosis of pancreatic cancer. Lancet Oncol 2009;10:88-95.

7 Bartosch-Harlid A, Andersson R: Diabetes mellitus in pancreatic cancer and the need for diagnosis of asymptomatic disease. Pancreatology 2010;10:423-428.

-8 Zhang HH, Guo XL: Combinational strategies of metformin and chemotherapy in cancers. Cancer Chemother Pharmacol 2016;78:13-26.

-9 Bhaw-Luximon A, Jhurry D: Metformin in pancreatic cancer treatment: from clinical trials through basic research to biomarker quantification. J Cancer Res Clin Oncol 2016;142:2159-2171.

10 De Souza A, Khawaja KI, Masud F, Saif MW: Metformin and pancreatic cancer: Is there a role? Cancer Chemother Pharmacol 2016;77:235-242.

11 Gandini S, Puntoni M, Heckman-Stoddard BM, Dunn BK, Ford L, DeCensi A, Szabo E: Metformin and cancer risk and mortality: a systematic review and meta-analysis taking into account biases and confounders. Cancer Prev Res (Phila) 2014;7:867-885.

12 Lega IC, Shah PS, Margel D, Beyene J, Rochon PA, Lipscombe LL: The effect of metformin on mortality following cancer among patients with diabetes. Cancer Epidemiol Biomarkers Prev 2014;23:1974-1984.

13 Coyle C, Cafferty FH, Vale C, Langley RE: Metformin as an adjuvant treatment for cancer: a systematic review and meta-analysis. Ann Oncol 2016;27:2184-2195.

14 Zhang ZJ, Li S: The prognostic value of metformin for cancer patients with concurrent diabetes: a systematic review and meta-analysis. Diabetes Obes Metab 2014;16:707-710.

15 Landman GW, Kleefstra N, van Hateren KJ, Groenier KH, Gans RO, Bilo HJ: Metformin associated with lower cancer mortality in type 2 diabetes: ZODIAC-16. Diabetes Care 2010;33:322-326.

16 Wang Z, Lai ST, Xie L, Zhao JD, Ma NY, Zhu J, Ren ZG, Jiang GL: Metformin is associated with reduced risk of pancreatic cancer in patients with type 2 diabetes mellitus: a systematic review and meta-analysis. Diabetes Res Clin Pract 2014;106:19-26.

17 Zakikhani M, Dowling R, Fantus IG, Sonenberg N, Pollak M: Metformin is an AMP kinase-dependent growth inhibitor for breast cancer cells. Cancer Res 2006;66:10269-10273.

18 Kim J, Kundu M, Viollet B, Guan KL: AMPK and mTOR regulate autophagy through direct phosphorylation of Ulk1. Nat Cell Biol 2011;13:132-141. 


\section{Cellular Physiology Cell Physiol Biochem 2018;49:837-847 and Biochemistry DOI: 10.1159/000493214 202018 The Author(s). Published by S. Karger AG, Basel

Wan et al.: Metformin and Pancreatic Cancer

19 Park DB: Metformin Promotes Apoptosis but Suppresses Autophagy in Glucose-Deprived H4IIE Hepatocellular Carcinoma Cells. Diabetes Metab J 2015;39:518-527.

20 Ben SI, Le Marchand-Brustel Y, Tanti JF, Bost F: Metformin in cancer therapy: a new perspective for an old antidiabetic drug? Mol Cancer Ther 2010;9:1092-1099.

-21 Ambe CM, Mahipal A, Fulp J, Chen L, Malafa MP: Effect of Metformin Use on Survival in Resectable Pancreatic Cancer: A Single-Institution Experience and Review of the Literature. PLoS One 2016;11:e151632.

-22 Zhang H, Gao C, Fang L, Zhao HC, Yao SK: Metformin and reduced risk of hepatocellular carcinoma in diabetic patients: a meta-analysis. Scand J Gastroenterol 2013;48:78-87.

-23 Stang A: Critical evaluation of the Newcastle-Ottawa scale for the assessment of the quality of nonrandomized studies in meta-analyses. Eur J Epidemiol 2010;25:603-605.

24 Parmar MK, Torri V, Stewart L: Extracting summary statistics to perform meta-analyses of the published literature for survival endpoints. Stat Med 1998;17:2815-2834.

25 Tierney JF, Stewart LA, Ghersi D, Burdett S, Sydes MR: Practical methods for incorporating summary timeto-event data into meta-analysis. Trials 2007;8:16.

26 Reni M, Dugnani E, Cereda S, Belli C, Balzano G, Nicoletti R, Liberati D, Pasquale V, Scavini M, Maggiora P, Sordi V, Lampasona V, Ceraulo D, Di Terlizzi G, Doglioni C, Falconi M, Piemonti L: (Ir)relevance of Metformin Treatment in Patients with Metastatic Pancreatic Cancer: An Open-Label, Randomized Phase II Trial. Clin Cancer Res 2016;22:1076-1085.

27 Lee SH, Yoon SH, Lee HS, Chung MJ, Park JY, Park SW, Song SY, Chung JB, Bang S: Can metformin change the prognosis of pancreatic cancer? Retrospective study for pancreatic cancer patients with pre-existing diabetes mellitus type 2. Dig Liver Dis 2016;48:435-440.

-28 Cheon YK, Koo JK, Lee YS, Lee TY, Shim CS: Elevated hemoglobin A1c levels are associated with worse survival in advanced pancreatic cancer patients with diabetes. Gut Liver 2014;8:205-214.

29 Hwang AL, Haynes K, Hwang WT, Yang YX: Metformin and survival in pancreatic cancer: a retrospective cohort study. Pancreas 2013;42:1054-1059.

-30 Amin S, Mhango G, Lin J, Aronson A, Wisnivesky J, Boffetta P, Lucas AL: Metformin Improves Survival in Patients with Pancreatic Ductal Adenocarcinoma and Pre-Existing Diabetes: A Propensity Score Analysis. Am J Gastroenterol 2016;111:1350-1357.

-31 Kordes S, Pollak MN, Zwinderman AH, Mathot RA, Weterman MJ, Beeker A, Punt CJ, Richel DJ, Wilmink JW: Metformin in patients with advanced pancreatic cancer: a double-blind, randomised, placebo-controlled phase 2 trial. Lancet Oncol 2015;16:839-847.

-32 Chaiteerakij R, Petersen GM, Bamlet WR, Chaffee KG, Zhen DB, Burch PA, Leof ER, Roberts LR, Oberg AL: Metformin Use and Survival of Patients With Pancreatic Cancer: A Cautionary Lesson. J Clin Oncol 2016;34:1898-1904.

33 Sadeghi N, Abbruzzese JL, Yeung SC, Hassan M, Li D: Metformin use is associated with better survival of diabetic patients with pancreatic cancer. Clin Cancer Res 2012;18:2905-2912.

-34 Cerullo M, Gani F, Chen SY, Canner J, Pawlik TM: Metformin Use Is Associated with Improved Survival in Patients Undergoing Resection for Pancreatic Cancer. J Gastrointest Surg 2016;20:1572-1580.

-35 Kozak MM, Anderson EM, von Eyben R, Pai JS, Poultsides GA, Visser BC, Norton JA, Koong AC, Chang DT: Statin and Metformin Use Prolongs Survival in Patients With Resectable Pancreatic Cancer. Pancreas 2016;45:64-70.

-36 Toomey P, Teta A, Patel K, Luberice K, Ross S, Rosemurgy A: Sulfonylureas (Not Metformin) Improve Survival for Patients with Diabetes and Resectable Pancreatic Adenocarcinoma. Pancreat Disord Ther 2015;5:S5.

-37 Choi Y, Kim TY, Oh DY, Lee KH, Han SW, Im SA, Kim TY, Bang YJ: The Impact of Diabetes Mellitus and Metformin Treatment on Survival of Patients with Advanced Pancreatic Cancer Undergoing Chemotherapy. Cancer Res Treat 2016;48:171-179.

38 Beg MS, Gupta A, Sher D, Ali S, Khan S, Gao A, Stewart T, Ahn C, Berry J,Mortensen EM: Impact of Concurrent Medication Use on Pancreatic Cancer Survival-SEER-Medicare Analysis. Am J Clin Oncol 2017; DOI:10.1097/COC.0000000000000359. 


\section{Cellular Physiology Cell Physiol Biochem 2018;49:837-847

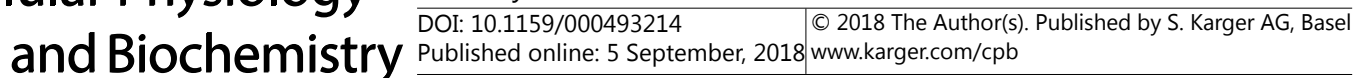 \\ Wan et al.: Metformin and Pancreatic Cancer}

-39 Jang WI, Kim MS, Kang SH, Jo AJ, Kim YJ, Tchoe HJ, Park CM, Kim HJ, Choi JA, Choi HJ, Paik EK, Seo YS, Yoo HJ, Kang JK, Han CJ, Kim YJ, Kim SB, Ko MJ: Association between metformin use and mortality in patients with type 2 diabetes mellitus and localized resectable pancreatic cancer: a nationwide population-based study in Korea. Oncotarget 2017;7;8:9587-9596.

-40 Frouws MA, Mulder BG, Bastiaannet E, Zanders MM, van Herk-Sukel MP, de Leede EM, Bonsing BA, Mieog JS, Van de Velde CJ, Liefers GJ: No association between metformin use and survival in patients with pancreatic cancer: An observational cohort study. Medicine (Baltimore) 2017;96:e6229.

41 E JY, Lu SE, Lin Y, Graber JM, Rotter D, Zhang L, Petersen GM, Demissie K, Lu-Yao G, Tan XL: Differential and Joint Effects of Metformin and Statins on Overall Survival of Elderly Patients with Pancreatic Adenocarcinoma: A Large Population-Based Study. Cancer Epidemiol Biomarkers Prev 2017;26:12251232.

42 Zhou DC, Gong H, Tan CQ Luo JQ: Prognostic significance of anti-diabetic medications in pancreatic cancer: A meta-analysis. Oncotarget 2017;8:62349-62357.

43 Dong YW, Shi YQ, He LW, Cui XY, Su PZ: Effects of metformin on survival outcomes of pancreatic cancer: a meta-analysis. Oncotarget 2017;8:55478-55488.

44 Li X, Li T, Liu Z, Gou S, Wang C: The effect of metformin on survival of patients with pancreatic cancer: a meta-analysis. Sci Rep 2017;7:5825.

45 Zhang JW, Sun Q: Metformin may improve the prognosis of patients with pancreatic cancer. Asian Pac J Cancer Prev 2015;16:3937-3940.

46 Zhou PT, Li B, Liu FR, Zhang MC, Wang Q, Li YY, Xu C, Liu YH, Yao Y, Li D: Metformin is associated with survival benefit in pancreatic cancer patients with diabetes: a systematic review and meta-analysis. Oncotarget 2017;8:25242-25250.

47 E J, Graber JM, Lu SE, Lin Y, Lu-Yao G, Tan XL: Effect of Metformin and Statin Use on Survival in Pancreatic Cancer Patients: a Systematic Literature Review and Meta-analysis. Curr Med Chem 2017; DOI:10.2174/09 29867324666170412145232.

48 Suissa S, Azoulay L: Metformin and the risk of cancer: time-related biases in observational studies. Diabetes Care 2012;35:2665-2673.

49 Monami M, Dicembrini I, Mannucci E: Thiazolidinediones and cancer: results of a meta-analysis of randomized clinical trials. Acta Diabetol 2014;51:91-101. 